\title{
Equilibria of Deferred Acceptance with Complete Lists*
}

\author{
Bettina Klaus $^{\dagger} \quad$ Flip Klijn ${ }^{\ddagger}$
}

April 4, 2016

\begin{abstract}
We study the structure of the set of (Nash) equilibria of a deferred acceptance game with complete lists: for a given marriage market with complete lists, men propose to women truthfully while women can accept or reject proposals strategically throughout the deferred-acceptance algorithm. Zhou (1991) studied this game and showed that a matching that is stable with respect to the true preferences can be supported by some preference profile (possibly a non-equilibrium one) if and only if it can be supported by an equilibrium as well. In particular, this result implies the existence of equilibria since the men-optimal stable matching is supported by true preferences and hence an equilibrium outcome. We answer an open question Zhou posed by showing that there need not exist an equilibrium matching that weakly dominates all other equilibrium matchings from the women's point of view (Theorem 2).
\end{abstract}

Keywords: matching, stability, complete lists, Nash equilibria.

JEL-Numbers: C72, C78, D47.

*We thank Battal Doğan for comments on a previous version.

${ }^{\dagger}$ Faculty of Business and Economics, University of Lausanne, Internef 538, CH-1015 Lausanne, Switzerland; e-mail: bettina.klaus@unil.ch. B. Klaus gratefully acknowledges financial support from the Swiss National Science Foundation (SNFS).

$\ddagger$ Corresponding author. Institute for Economic Analysis (CSIC) and Barcelona GSE, Campus UAB, 08193 Bellaterra (Barcelona), Spain; e-mail: flip.klijn@iae.csic.es. F. Klijn gratefully acknowledges financial support from the Generalitat de Catalunya (2014-SGR-1064), the Spanish Ministry of Economy and Competitiveness through Plan Nacional I+D+i (ECO2014-59302-P), and the Severo Ochoa Programme for Centres of Excellence in R\&D (SEV-2015-0563).

(C) 2016. This manuscript version is made available under the Elsevier user license http://www.elsevier.com/open-access/userlicense/1.0/ 


\section{Introduction}

The so-called marriage model is concerned with (two-sided, one-to-one) matching markets where the two sides of the market are, for instance, men and women (or firms and workers). A matching is then a partition of all men and women into couples and unmatched agents. Such a matching is "stable" if each man and woman has an acceptable match, and no man and woman prefer one another to their respective matches. Gale and Shapley (1962) were the first to formalize this notion of stability and presented their deferred-acceptance algorithm to calculate stable matchings. Stability proved to be an essential property in several real-life markets. For instance, in many centralized labor markets, clearinghouses are most often successful if they produce stable matchings. ${ }^{1}$ This inspired many researchers to study stability; see Roth and Sotomayor (1990) for a classic survey, and Vulkan et al. (2013) and Manlove (2013) for more recent surveys.

In the original Gale-Shapley model, the preference lists of the agents are assumed to be complete, i.e., for each agent all agents on the other side are acceptable. In other words, remaining unmatched is not desirable. Moreover, agents cannot be (falsely) declared unacceptable. The men-proposing deferred-acceptance algorithm with complete lists induces a game where it is a weakly dominant strategy for each man to reveal his preferences truthfully (Dubins and Freedman, 1981; Roth, 1982). For marriage markets with complete lists, Roth (1984b) showed that the matching induced by any equilibrium in (weakly) undominated strategies is stable with respect to the true preferences. Zhou (1991) assumed that men propose to women truthfully while women can accept or reject proposals strategically throughout the deferred-acceptance algorithm and showed that a matching that is stable with respect to the true preferences can be supported by some preference profile (possibly a non-equilibrium one) if and only if it can be supported by an equilibrium as well. In particular, this result implies the existence of equilibria since the men-optimal stable matching is supported by true preferences and hence an equilibrium outcome. Zhou (1991) conjectured the existence of an equilibrium matching (possibly not the women-optimal stable matching) that weakly dominates all other equilibrium matchings from the women's point of view. Teo et al. (2001) showed that it is not always possible for a woman $w$ to obtain her women-optimal stable partner from the men-proposing deferred-acceptance algorithm, but they assumed that woman $w$ is the only agent acting strategically, i.e., their study does not consider the equilibria of the deferred-acceptance game.

In this note, we solve Zhou's (1991) conjecture in the negative by showing that there need not exist an equilibrium matching (the women-optimal stable matching or not) that weakly dominates all other equilibrium matchings from the women's point of view (Theorem 2). We complement Zhou's and our findings by showing that the set of equilibrium matchings also need not be "connected." More precisely, suppose two stable matchings are supported by equilibria. Then, it can happen that any stable matching that is located between the two stable matchings (in terms of each agent's preferences) is not supported

\footnotetext{
${ }^{1}$ See Roth (1984a) and Roth and Xing (1994) for empirical evidence.
} 
by an equilibrium.

The results for the original Gale-Shapley model contrast with those for the model that allows for "rejections," i.e., an agent's preference list need not be complete in the sense that some agents on the other side of the market may be unacceptable. In this case, agents have the option of declaring some agents as unacceptable. In the game induced by the men-proposing deferred-acceptance algorithm, the set of equilibrium outcomes coincides with the set of stable matchings (Gale and Sotomayor, 1985; Roth, 1984b). An important consequence is that the remarkable properties of the set of stable matchings carry over to the set of equilibrium outcomes. In particular, there exist an equilibrium matching (the women-optimal stable matching) that weakly dominates all other equilibrium matchings from the women's point of view. However, for the more general many-to-one matching model such an equilibrium matching also does not need to exist (Jaramillo et al., 2013, Example 1).

\section{Model}

There are two finite and disjoint sets of agents: a set $M=\left\{m_{1}, \ldots, m_{n}\right\}$ of men and a set $W=\left\{w_{1}, \ldots, w_{n}\right\}$ of women. Thus, $|M \cup W|=2 n$. We denote a generic agent by $i$, a generic man by $m$, and a generic woman by $w$.

Each agent has a complete, transitive, and strict preference relation over the agents on the other side of the market. Hence, man $m$ 's preferences $\succ_{m}$ can be represented as a strict ordering $P_{m}$ of the elements in $W$, for instance: $P_{m}=w_{3} w_{4} w_{2} w_{1}$ which indicates that $m$ prefers $w_{3}$ to $w_{4}$ to $w_{2}$ to $w_{1}$. Similarly, woman $w^{\prime}$ 's preferences $\succ_{w}$ can be represented as a strict ordering $P_{w}$ of the elements in $M$. For any $i \in M \cup W$, we write $j \succeq_{i} k$ if $j \succ_{i} k$ or $j=k$. For any $I \subseteq M \cup W$, we define $P_{I} \equiv\left(P_{i}\right)_{i \in I}$. We write $P$ instead of $P_{M \cup W}$.

A matching market is a triple $(M, W, P)$, or shortly $P$. A matching for $(M, W, P)$ is a function $\mu: M \cup W \rightarrow M \cup W$ such that for all $m \in M$ and $w \in W$ it holds that $\mu(m)=w \Leftrightarrow \mu(w)=m$. If $\mu(m)=w$, then man $m$ and woman $w$ are matched to one another. We call $\mu(i)$ the match of agent $i$ at $\mu$. When denoting a matching $\mu$ we list the women that are matched to men $m_{1}, \ldots, m_{n}$; e.g., $\mu=w_{3}, w_{4}, w_{2}, w_{1}$ denotes a matching where $m_{1}$ is matched to $w_{3}, m_{2}$ to $w_{4}, m_{3}$ to $w_{2}$, and $m_{4}$ to $w_{1}$. Alternatively, a matching can be described by the list of men that are matched to women $w_{1}, \ldots, w_{n}$.

A key property of matchings is stability. If an agent can improve upon its present match by switching to another agent such that this agent is better off as well, then this blocking clearly would cause instability. For a given matching $\mu$, a man $m$ and a woman $w$ are a blocking pair if they are not matched to one another but prefer one another to their current match at $\mu$, i.e., $w \succ_{m} \mu(m)$ and $m \succ_{w} \mu(w)$. A matching is stable if there are no blocking pairs. We denote the set of stable matchings for matching market $P$ by $\mathcal{S}(P)$. Gale and Shapley (1962) proved that $\mathcal{S}(P) \neq \emptyset$.

In fact, Gale and Shapley (1962) showed that there is a men-optimal (womenpessimal) stable matching $\boldsymbol{\mu}_{\boldsymbol{M}}^{\boldsymbol{P}}$, i.e., for each man $m \in M$, each woman $w \in W$, 
and each stable matching $\mu, \mu_{M}^{P}(m) \succeq_{m} \mu(m)$ and $\mu(w) \succeq_{w} \mu_{M}^{P}(w)$. Similarly, there is a men-pessimal (women-optimal) stable matching $\boldsymbol{\mu}_{\boldsymbol{W}}^{\boldsymbol{P}}$. Gale and Shapley (1962) provided an algorithm, called the deferred-acceptance algorithm, to compute $\mu_{M}^{P}$ and $\mu_{W}^{P}$. Next, we describe the (men-proposing) deferred-acceptance (DA) algorithm ${ }^{2}$ to obtain the men-optimal stable matching $\mu_{M}^{P} \in \mathcal{S}(P)$ for any preference profile $P$.

\section{DA algorithm:}

STEP 1. Each man proposes to his most preferred woman. Each woman who receives at least one proposal is tentatively matched to her most preferred proposer and rejects all other proposers.

STEP $k \geq 2$. Each man who has been rejected in Step $k-1$ proposes to his most preferred woman among the ones that have not rejected him yet. Each woman who receives at least one proposal is tentatively matched to her most preferred man among the ones that proposed to her and the one she is currently tentatively matched to (if any)- all other proposers are rejected.

The algorithm terminates when no man is rejected. Then, tentative matches become final, and the resulting matching is $\mu_{M}^{P}$. Let $\varphi$ be the function that associates each preference profile $P$ with the men-optimal stable matching $\mu_{M}^{P}$ for $P$, i.e., $\varphi(P)=\mu_{M}^{P}$.

The DA algorithm induces a game form where each agent can reveal a preference relation over the other side of the market. For each strategy profile $Q$, the DA algorithm produces $\varphi(Q)$ as the outcome. With each true preference profile $P$, the DA algorithm induces a game $\boldsymbol{\Gamma}(\boldsymbol{P})$. Dubins and Freedman (1981) and Roth (1982) showed that for each man $m \in M$ it is a weakly dominant strategy to play $P_{m}$, i.e., to reveal his preferences truthfully. Henceforth, we will assume that men always state the truth and that women are the only strategic agents.

Roth (1984b) showed that for any strategy profile $Q$ that is a Nash equilibrium (in weakly undominated strategies) of the game $\Gamma(P)$, matching $\varphi(Q)$ is stable with respect to the true preferences $P$. Zhou (1991) proved the existence of Nash equilibria of $\Gamma(P)$ and characterized the matchings that can be sustained at Nash equilibria. Let $\mathcal{E}(P)$ be the matchings that are the outcome of some Nash equilibrium of $\Gamma(P)$. Let $\mathcal{O}\left(P_{M}\right)$ be the matchings that can be obtained at some strategy profile where men state the truth and women play any set of strategies (which do not necessarily constitute a Nash equilibrium), i.e.,

$$
\mathcal{O}\left(P_{M}\right)=\left\{\varphi\left(P_{M}, Q_{W}\right): Q_{W} \text { is a strategy profile of the women }\right\} .
$$

Theorem 1. [Zhou, 1991, Theorem 1]

For each market $P, \mathcal{E}(P)=\mathcal{S}(P) \cap \mathcal{O}\left(P_{M}\right)$. In particular, since $\mu_{M}^{P}=\varphi\left(P_{M}, P_{W}\right) \in \mathcal{S}(P)$, $\mathcal{E}(P) \neq \emptyset$.

Zhou (1991, Theorem 2) also showed that $\mathcal{E}(P)$ contains matchings different from $\mu_{M}^{P}$ if truth-telling is not an equilibrium profile for women.

\footnotetext{
${ }^{2}$ By switching the roles of men and women in the deferred-acceptance algorithm, matching $\mu_{W}^{P}$ is obtained.
} 


\section{Results}

Zhou (1991, p. 29) asked whether women can coordinate their manipulations in an optimal fashion. More precisely, "does there exist a matching [in] $\mathcal{E}(P)$ that weakly dominates all other matchings in $\mathcal{E}(P)$ from the women's point of view? Such a matching, if [it] exists, seems more likely to emerge than others. ... We leave it as a conjecture for future research."

The next theorem answers Zhou's (1991) question.

Theorem 2. If $n \leq 3$, then for any market $P$ there exists a matching $\mu \in \mathcal{E}(P)$ that weakly dominates all other matchings $\nu \in \mathcal{E}(P)$ from the women's point of view, i.e., for all $w \in W, \mu(w) \succeq_{w} \nu(w)$. If $n \geq 4$, this is not necessarily true.

Proof. Recall that by Theorem 1, for all markets $P, \mathcal{E}(P) \subseteq \mathcal{S}(P)$.

Let $n \leq 2$. Then, for any market $P$ there are at most 2 stable matchings, and hence the statement is trivially true.

Let $n=3$. Let $P$ be a market. Suppose by contradiction that there exists no matching in $\mathcal{E}(P)$ that weakly dominates all other matchings in $\mathcal{E}(P)$ from the women's point of view. Then, women cannot find a jointly optimal equilibrium matching and hence there exist at least two distinct (stable) matchings $\mu^{\prime}, \mu^{\prime \prime} \in \mathcal{E}(P)$, each of which is considered optimal by some women but not others. Thus, there are distinct women, say $w_{1}$ and $w_{2}$, such that woman $w_{1}$ finds $\mu^{\prime}$ optimal but not $\mu^{\prime \prime}\left[\right.$ for all $\nu \in \mathcal{E}(P), \mu^{\prime}\left(w_{1}\right) \succeq_{w_{1}} \nu\left(w_{1}\right)$ and $\mu^{\prime}\left(w_{1}\right) \succ_{w_{1}} \mu^{\prime \prime}\left(w_{1}\right)$ ] while woman $w_{2}$ finds $\mu^{\prime \prime}$ optimal but not $\mu^{\prime}$ [for all $\nu \in \mathcal{E}(P)$, $\mu^{\prime \prime}\left(w_{2}\right) \succeq_{w_{2}} \nu\left(w_{2}\right)$ and $\left.\mu^{\prime \prime}\left(w_{2}\right) \succ_{w_{2}} \mu^{\prime}\left(w_{2}\right)\right]$.

Let $W\left(\mu^{\prime}\right)$ be the set of women who strictly prefer $\mu^{\prime}$ to $\mu^{\prime \prime}$ and $M\left(\mu^{\prime}\right)$ be the set of men who strictly prefer $\mu^{\prime}$ to $\mu^{\prime \prime}$. Analogously define $W\left(\mu^{\prime \prime}\right)$ and $M\left(\mu^{\prime \prime}\right)$. Since $\mu^{\prime}, \mu^{\prime \prime} \in$ $\mathcal{E}(P) \subseteq \mathcal{S}(P)$ it follows from Donald Knuth's decomposition lemma (Roth and Sotomayor, 1990, Corollary 2.21) that for all $w \in W,\left[w \in W\left(\mu^{\prime}\right)\right.$ if and only if $\left.\mu^{\prime}(w) \in M\left(\mu^{\prime \prime}\right)\right]$ and $\left[w \in W\left(\mu^{\prime \prime}\right)\right.$ if and only if $\left.\mu^{\prime \prime}(w) \in M\left(\mu^{\prime}\right)\right]$.

Note that $w_{1} \in W\left(\mu^{\prime}\right)$ and $w_{2} \in W\left(\mu^{\prime \prime}\right)$. Then, $w_{1} \in W\left(\mu^{\prime}\right)$ implies $m^{\prime} \equiv \mu^{\prime}\left(w_{1}\right) \in$ $M\left(\mu^{\prime \prime}\right)$. By assumption, $w_{1}$ gets different matches at $\mu^{\prime}$ and $\mu^{\prime \prime}$. Hence, $m^{\prime}=\mu^{\prime}\left(w_{1}\right)$ gets different matches at $\mu^{\prime}$ and $\mu^{\prime \prime}$. But then also $w^{\prime \prime} \equiv \mu^{\prime \prime}\left(m^{\prime}\right)$ gets different matches at $\mu^{\prime}$ and $\mu^{\prime \prime}$. Hence, $w^{\prime \prime} \in W\left(\mu^{\prime}\right) \cup W\left(\mu^{\prime \prime}\right)$. Suppose $w^{\prime \prime} \in W\left(\mu^{\prime \prime}\right)$. Since $m^{\prime} \in M\left(\mu^{\prime \prime}\right)$ and $w^{\prime \prime}=\mu^{\prime \prime}\left(m^{\prime}\right),\left(m^{\prime}, w^{\prime \prime}\right)$ is a blocking pair for $\mu^{\prime}$, which contradicts the stability of $\mu^{\prime}$. Hence, $w^{\prime \prime} \in W\left(\mu^{\prime}\right)$. Since (i) $\mu^{\prime}\left(m^{\prime}\right)=w_{1}$ and $\mu^{\prime \prime}\left(m^{\prime}\right)=w^{\prime \prime}$ and (ii) $m^{\prime}$ gets different matches at $\mu^{\prime}$ and $\mu^{\prime \prime}$, it follows that $w_{1} \neq w^{\prime \prime}$. Hence, $\left|W\left(\mu^{\prime}\right)\right| \geq 2$. Similarly, it follows that $w_{2} \in W\left(\mu^{\prime \prime}\right)$ implies $\left|W\left(\mu^{\prime \prime}\right)\right| \geq 2$. Since $W\left(\mu^{\prime}\right) \cap W\left(\mu^{\prime \prime}\right)=\emptyset$, it follows that $|W| \geq\left|W\left(\mu^{\prime}\right)\right|+\left|W\left(\mu^{\prime \prime}\right)\right| \geq 4$, which contradicts $|W|=n=3$.

If $n=4$, then Example 1 shows that there need not exist an equilibrium matching that weakly dominates all other equilibrium matchings from the women's point of view. The example extends to the case $n>4$ by making for each $l>4$, the members of $\left(m_{l}, w_{l}\right)$ each other's mutually best possible partner. 
Example 1. [No optimal equilibrium outcome for the women] Consider the matching market $(M, W, P)$ where $M=\left\{m_{1}, m_{2}, m_{3}, m_{4}\right\}, W=\left\{w_{1}, w_{2}, w_{3}\right.$, $\left.w_{4}\right\}$, and preferences $P$ given by Table 1 . In each column, higher placed agents are more preferred agents.

\begin{tabular}{|c|c|c|c|c|c|c|c|}
\hline \multicolumn{4}{|c|}{ men } & \multicolumn{4}{|c|}{ women } \\
\hline$m_{1}$ & $m_{2}$ & $m_{3}$ & $m_{4}$ & $w_{1}$ & $w_{2}$ & $w_{3}$ & $w_{4}$ \\
\hline$w_{1}$ & $w_{4}$ & $w_{1}$ & $w_{4}$ & $m_{2}$ & $m_{1}$ & $m_{4}$ & $m_{3}$ \\
\hline$w_{2}$ & $w_{2}$ & $w_{3}$ & $w_{3}$ & $m_{1}$ & $m_{2}$ & $m_{3}$ & $m_{4}$ \\
\hline \multirow[t]{2}{*}{$\cdots$} & $w_{1}$ & $w_{4}$ & $\cdots$ & $\ldots$ & $\ldots$ & $\ldots$ & $\ldots$ \\
\hline & $w_{3}$ & $w_{3}$ & & & & & \\
\hline
\end{tabular}

Table 1: Preferences $P$ in Example 1

The entries $\cdots$ can be any agents as long as each column is a preference relation over the agents on the other side of the market. The set of stable matchings, given by $\mathcal{S}(P)=\left\{\mu_{M}^{P}=\mu_{1}, \mu_{2}, \mu_{3}, \mu_{4}=\mu_{W}^{P}\right\}$, is depicted in Table $2 .^{3}$ In all tables, the menoptimal stable matching is depicted in boldface while the women-optimal stable matching is the boxed matching.

\begin{tabular}{|c|c|c|c|c|c|c|c|c|}
\hline \multirow[b]{3}{*}{$\mu_{M}^{P}=\mu_{1}:$} & \multicolumn{4}{|c|}{ men } & \multicolumn{4}{|c|}{ women } \\
\hline & $m_{1}$ & $m_{2}$ & $m_{3}$ & $m_{4}$ & $w_{1}$ & $w_{2}$ & $w_{3}$ & $w_{4}$ \\
\hline & $w_{1}$ & $w_{2}$ & $w_{3}$ & $w_{4}$ & $m_{1}$ & $m_{2}$ & $m_{3}$ & $m_{4}$ \\
\hline$\mu_{2}:$ & $w_{2}$ & $w_{1}$ & $w_{3}$ & $w_{4}$ & $m_{2}$ & $m_{1}$ & $m_{3}$ & $m_{4}$ \\
\hline$\mu_{3}:$ & $w_{1}$ & $w_{2}$ & $w_{4}$ & $w_{3}$ & $m_{1}$ & $m_{2}$ & $m_{4}$ & $m_{3}$ \\
\hline$\mu_{W}^{P}=\mu_{4}:$ & $w_{2}$ & $w_{1}$ & $w_{4}$ & $w_{3}$ & $m_{2}$ & $m_{1}$ & $m_{4}$ & $m_{3}$ \\
\hline
\end{tabular}

Table 2: The four stable matchings in Example 1

To show that there is no optimal equilibrium outcome for the women it is sufficient to prove that $\mathcal{E}(P)=\left\{\mu_{1}, \mu_{2}, \mu_{3}\right\}$ : for all women $w \in\left\{w_{1}, w_{2}\right\}, \mu_{2}(w) \succ_{w} \mu_{1}(w)=\mu_{3}(w)$, while for all women $w \in\left\{w_{3}, w_{4}\right\}, \mu_{3}(w) \succ_{w} \mu_{1}(w)=\mu_{2}(w)$.

We first show that $\mathcal{E}(P) \supseteq\left\{\mu_{1}, \mu_{2}, \mu_{3}\right\}$. By Theorem $1, \mu_{1}=\mu_{M}^{P} \in \mathcal{E}(P)$. Next, note that for profile $Q^{2} \equiv\left(P_{M}, Q_{W}^{2}\right)$, where $Q_{W}^{2}$ are the women's preferences given in Table 3 , we have $\varphi\left(Q^{2}\right)=\mu_{2}$. Hence, $\mu_{2} \in \mathcal{O}\left(P_{M}\right)$. So, by Theorem $1, \mu_{2} \in \mathcal{E}(P)$. Similarly, for profile $Q^{3} \equiv\left(P_{M}, Q_{W}^{3}\right)$, where $Q_{W}^{3}$ are the women's preferences given in Table 4 , we have $\varphi\left(Q^{3}\right)=\mu_{3}$, and hence, $\mu_{3} \in \mathcal{E}(P)$.

\footnotetext{
${ }^{3}$ It is easy to see that the set of stable matchings is indeed $\mathcal{S}(P)=\left\{\mu_{M}^{P}=\mu_{1}, \mu_{2}, \mu_{3}, \mu_{4}=\mu_{W}^{P}\right\}$. First, one computes $\mu_{M}^{P}$ and $\mu_{W}^{P}$ using the two versions of the DA algorithm. Second, one verifies that the only other two matchings $\mu$ with $\mu_{M}^{P}(m) \succeq_{m} \mu(m) \succeq_{m} \mu_{W}^{P}(m)$ for all $m \in M$ are $\mu_{2}$ and $\mu_{3}$. Finally, one checks the stability of $\mu_{2}$ and $\mu_{3}$.
} 


\begin{tabular}{cccc}
\hline \hline \multicolumn{4}{c}{ women } \\
\hline$w_{1}$ & $w_{2}$ & $w_{3}$ & $w_{4}$ \\
\hline$m_{2}$ & $m_{1}$ & $m_{4}$ & $m_{3}$ \\
$m_{3}$ & $m_{2}$ & $m_{3}$ & $m_{4}$ \\
$m_{1}$ & $\ldots$ & $\ldots$ & $\ldots$ \\
$m_{4}$ & & & \\
\hline
\end{tabular}

Table 3: Preferences $Q_{W}^{2}$

\begin{tabular}{cccc}
\hline \hline \multicolumn{4}{c}{ women } \\
\hline$w_{1}$ & $w_{2}$ & $w_{3}$ & $w_{4}$ \\
\hline$m_{2}$ & $m_{1}$ & $m_{4}$ & $m_{3}$ \\
$m_{1}$ & $m_{2}$ & $m_{3}$ & $m_{2}$ \\
$\ldots$ & $\ldots$ & $\ldots$ & $m_{4}$ \\
& & & $m_{1}$ \\
\hline \hline
\end{tabular}

Table 4: Preferences $Q_{W}^{3}$

Next, we show that $\mathcal{E}(P) \subseteq\left\{\mu_{1}, \mu_{2}, \mu_{3}\right\}$. By Theorem $1, \mathcal{E}(P)=\mathcal{S}(P) \cap \mathcal{O}\left(P_{M}\right) \subseteq$ $\left\{\mu_{1}, \mu_{2}, \mu_{3}, \mu_{4}\right\}$. Hence, it suffices to show that $\mu_{4} \notin \mathcal{E}(P)$. Since $\mu_{4} \in \mathcal{S}(P)$, we have to prove that $\mu_{4} \notin \mathcal{O}\left(P_{M}\right)$, i.e., for all possible preference profiles $Q_{W}$ of the women, $\varphi\left(P_{M}, Q_{W}\right) \neq \mu_{4}$. Let $Q_{W}$ be a preference profile of the women. Notice that in Step 1 of the DA algorithm, men $m_{1}$ and $m_{3}$ propose to woman $w_{1}$ and men $m_{2}$ and $m_{4}$ propose to woman $w_{4}$. Since each of these women has to reject one applicant, we distinguish between the four cases $^{4}$ depicted in the first column of Table 5. In all cases, the DA algorithm terminates in Step 2, i.e., the other two women receive exactly one proposal that they have to accept.

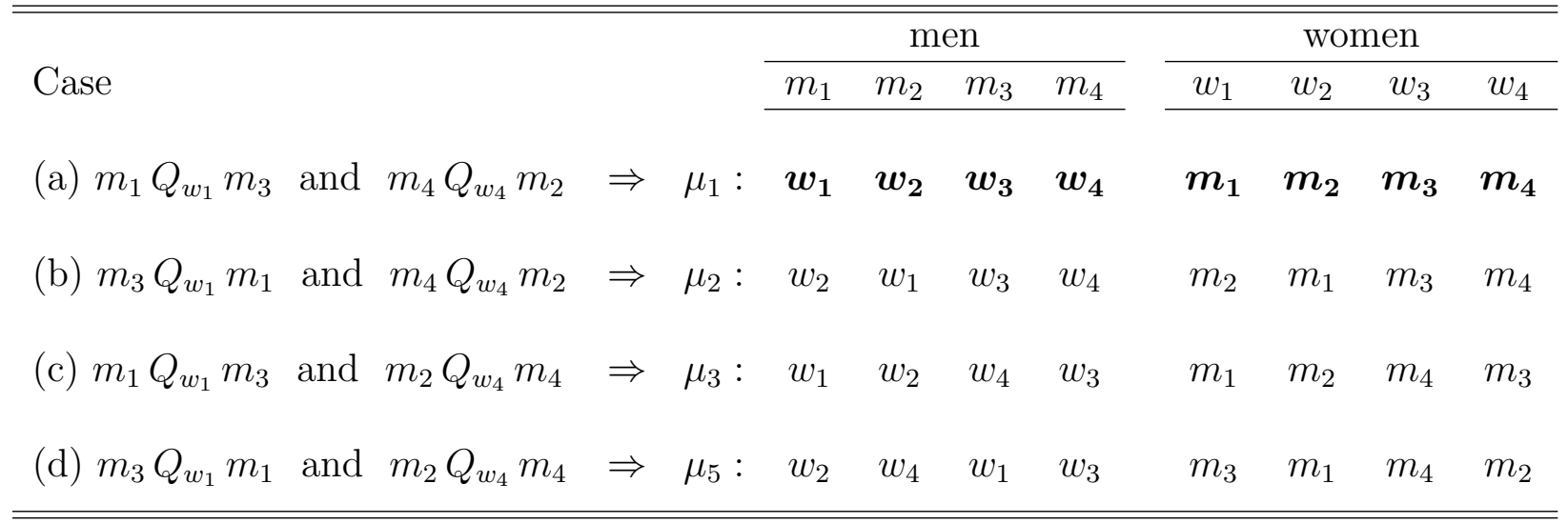

Table 5: The four outcomes $\varphi\left(P_{M}, Q_{W}\right)$ in Example 1

By applying the men-proposing DA algorithm, one easily verifies that in each case the resulting matching $\varphi\left(P_{M}, Q_{W}\right)$ is the one denoted in the same row. Since in all four cases $\varphi\left(P_{M}, Q_{W}\right) \neq \mu_{4}$ (note that matching $\mu_{5}$ is not stable), the proof is completed.

Our Example 1 and Example 1 in Zhou (1991) may suggest that for any market $P$ the set of equilibria is "connected," i.e., if $\mu, \mu^{\prime} \in \mathcal{E}(P)$, then for any $\nu \in \mathcal{S}(P)$ with $\mu(m) \succeq_{m} \nu(m) \succeq_{m} \mu^{\prime}(m)$ for all $m \in M, \nu \in \mathcal{E}(P)$. In Klaus and Klijn (2016, Example 2) we show that this need not be the case.

\footnotetext{
${ }^{4}$ For instance, $m_{1} Q_{w_{1}} m_{3}$ (in Cases (a) and (c)) means that in $w_{1}$ 's strategy (list) man $m_{1}$ is more preferred to $m_{3}$ and hence she rejects $m_{3}$ at Step 1 of the DA algorithm.
} 


\section{References}

Dubins, L. E. and Freedman, D. A. (1981): "Machiavelli and the Gale-Shapley Algorithm." American Mathematical Monthly, 88: 485-494.

Gale, D. and Shapley, L. S. (1962): "College Admissions and the Stability of Marriage." American Mathematical Monthly, 69: 9-15.

Gale, D. and Sotomayor, M. A. O. (1985): "Ms Machiavelli and the Stable Matching Problem." American Mathematical Monthly, 92: 261-268.

Jaramillo, P., Kayı, Ç., and Klijn, F. (2013): "Equilibria under Deferred Acceptance: Dropping Strategies, Filled Positions, and Welfare." Games and Economic Behavior, 82: 693-701.

Klaus, B. and Klijn, F. (2016): "Equilibria of Deferred Acceptance with Complete Lists." Cahier de recherches économiques du DEEP No. 16.08, Université de Lausanne.

Manlove, D. (2013): Algorithmics of Matching under Preferences. World Scientific Publishing.

Roth, A. E. (1982): "The Economics of Matching: Stability and Incentives." Mathematics of Operations Research, 7: 617-628.

Roth, A. E. (1984a): "The Evolution of the Labor Market for Medical Interns and Residents: A Case Study in Game Theory." Journal of Political Economy, 92: 991-1016.

Roth, A. E. (1984b): "Misrepresentation and Stability in the Marriage Problem." Journal of Economic Theory, 34: 383-387.

Roth, A. E. and Sotomayor, M. A. O. (1990): Two-Sided Matching: A Study in GameTheoretic Modeling and Analysis. Cambridge University Press, Cambridge.

Roth, A. E. and Xing, X. (1994): "Jumping the Gun: Imperfections and Institutions related to the Timing of Market Transactions." American Economic Review, 84: 9921044.

Teo, C. P., Sethuraman, J., and Tan, W. P. (2001): "Gale-Shapley Stable Marriage Problem Revisited: Strategic Issues and Applications." Management Science, 47: 12521267.

Vulkan, N., Roth, A. E., and Neeman, Z., editors (2013): The Handbook of Market Design. Oxford University Press.

Zhou, L. (1991): "Stable Matchings and Equilibrium Outcomes of the Gale-Shapley's Algorithm for the Marriage Problem." Economics Letters, 36: 25-29. 